九州大学学術情報リポジトリ

Kyushu University Institutional Repository

\title{
Estimation of Coda-Wave Attenuation in the Vicinity of Metropolitan Cairo, Egypt
}

Moustafa, Sayed S.R

Egyptian National Network Laboratory, National Research Institute of Astronomy and Geophysics

Takenaka, Hiroshi

Department of Earth and Planetary Sciences, Faculty of Sciences, Kyushu University

Mamada, Yutaka

Institute of Geoscience, Geological Survey of Japan, National Institute of Advanced Industrial Science and Technology

https://doi.org/10.5109/1546837

出版情報: 九州大学大学院理学研究院紀要 : Series D, Earth and planetary sciences. 31 (2)， pp.71-79, 2002-02-07. Faculty of Science, Kyushu University

バージョン :

権利関係 : 
Mem. Fac. Sci., Kyushu Univ., Ser. D, Earth \& Planet. Sci., Vol. XXXI, No. 2, pp. 71-79, text-figs. 1-4, table 1, February 7, 2002

\title{
Estimation of Coda-Wave Attenuation in the Vicinity of Metropolitan Cairo, Egypt
}

\author{
Sayed S. R. MousTAFA*, Hiroshi TAKENAKA ${ }^{* *}$ and Yutaka MAMADA ${ }^{* * *}$
}

\begin{abstract}
Seismic wave attenuation in the shallow crust around Cairo, Egypt was investigated using seismograms of local earthquakes recorded by the Egyptian National Network (ENN). The seismic wave attenuation was measured in the study area from the time decay of coda wave amplitudes on narrow bandpass filtered seismograms on the basis of the single scattering theory. The frequency bands of interest are from $1.5 \mathrm{~Hz}$ to $18 \mathrm{~Hz}$. A regional attenuation law $Q_{\mathrm{c}}=104.66 f^{0.79}$ was obtained, with no significant differences throughout the region.
\end{abstract}

Keywords: Attenuation, coda wave, Cairo, Egypt , $Q_{0}$, seismic wave

\section{I . Introduction}

Seismic wave attenuation is one of significant factors that affect seismic ground motion. The disastrous earthquakes occurring in and/or around Egypt during the past decades, confirmed the stressing need for vigorous investigations to reduce the losses of lives and properties. Consequently, regional as well as detailed metropolitan planning in a seismic active area should rely on the proper awareness of a lot of different implications (including seismological) of the earthquakes in that region. The conclusions drawn from such studies must be combined in order to contribute to the mitigation of seismic hazard, especially in large urban areas where the social and economic cost can be significant. For that reason, the present study attempts to evaluate the response of ground formations to seismic motion at selected sites in the vicinity of Cairo metropolitan area that was struck by a strong earthquake $\left(M_{\mathrm{b}}=5.8\right)$ on October 12th, 1992 at 13:09:59.2 GMT. As a result of this disastrous event, 5004 houses were destroyed and 11540 slightly damaged, while more than 40000 people remained homeless. 552 people were killed and more than 9929 were injured.

Besides its societal importance, the 1992 earthquake attracts attention also because it occurred in one of very few tectonically quiet parts of Egypt. The epicentral area of 1992 earthquake, southwest of Cairo, and its surroundings are considered as a separate seismic zone in the general

\footnotetext{
Manuscript received September 13, 2001 ; accepted November 9, 2001.

*Egyptian National Network Laboratory, National Research Institute of Astronomy and Geophysics, Helwan, Cairo, Egypt.

** Department of Earth and Planetary Sciences, Faculty of Sciences, Kyushu University, Hakozaki, Fukuoka, Japan.

*** Institute of Geoscience, Geological Survey of Japan, National Institute of Advanced Industrial Science and Technology, Tsukuba, Japan.
} 
division of the active seismic zones in Egypt, depending on the epicentral distribution, seismicity level, and the similarity of focal mechanisms. Tectonically, the faults of this area are trending eastwest, parallel to the Mediterranean trend, or northwest-southwest, parallel to the Gulf of Suez trend. These directions accord with the surface features that appeared immediately after the occurrence of 1992 earthquake. Figure 1 shows the general recent seismicity pattern in Egypt as recorded by the Egyptian National Network ( $E N N$ ) for the period from 1997 to 2000.

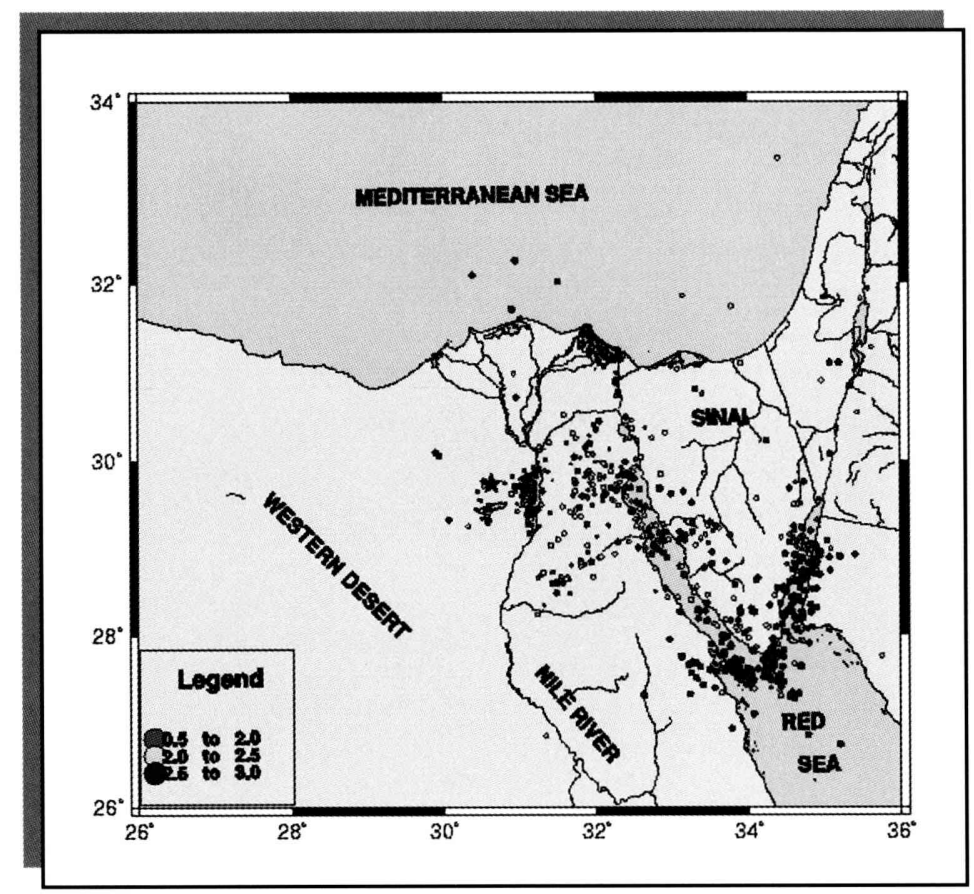

Fig. 1. Recent seismicity map of Egypt. Star is the epicenter of the 1992 earthquake.

There are some reasons which have motivated us to carry out the present study in order to quantify the general attenuation law for these vital area in Egypt. First, the extensive devastation during the 1992 catastrophic earthquake requires vigorous investigations to reduce the losses of lives and properties. As well as, lack of mathematical formulas that pay attention to local geological conditions to be used in the prediction of ground motion response and the absence of a good definition and estimation of seismic attenuation law for rays propagating in this area.

The main goal of the current study is to evaluate the attenuation of seismic shear waves in the vicinity of Cairo metropolitan area, incorporating the evaluation of the quality factor of coda waves as a function of frequency.

\section{Data}

The data used in this study are velocity seismograms of events occurring within or around the study area, which were recorded by three-component seismometers connected to digital data acquisition systems at three stations (HAG, FYM, and KOT) of the ENN. We selected 35 
seismograms with high $S / N$ ratio from local earthquakes recorded in the period from October 1997 to July 2000. The magnitude is less than three for the most part. Figure 2 shows the location of the three seismic stations together with the location of the events used in this study. The hypocentral locations were determined by the $E N N$. The focal depths and epicentral distances of these events are estimated to be less than $20 \mathrm{~km}$ and $40 \mathrm{~km}$ respectively by assuming a homogeneous velocity structure with $P$ - and $S$-wave velocities of $5.5 \mathrm{~km} / \mathrm{s}$ and $3.2 \mathrm{~km} / \mathrm{s}$, respectively. Direct body waves are thus expected to penetrate only the upper shallow part of the crust.

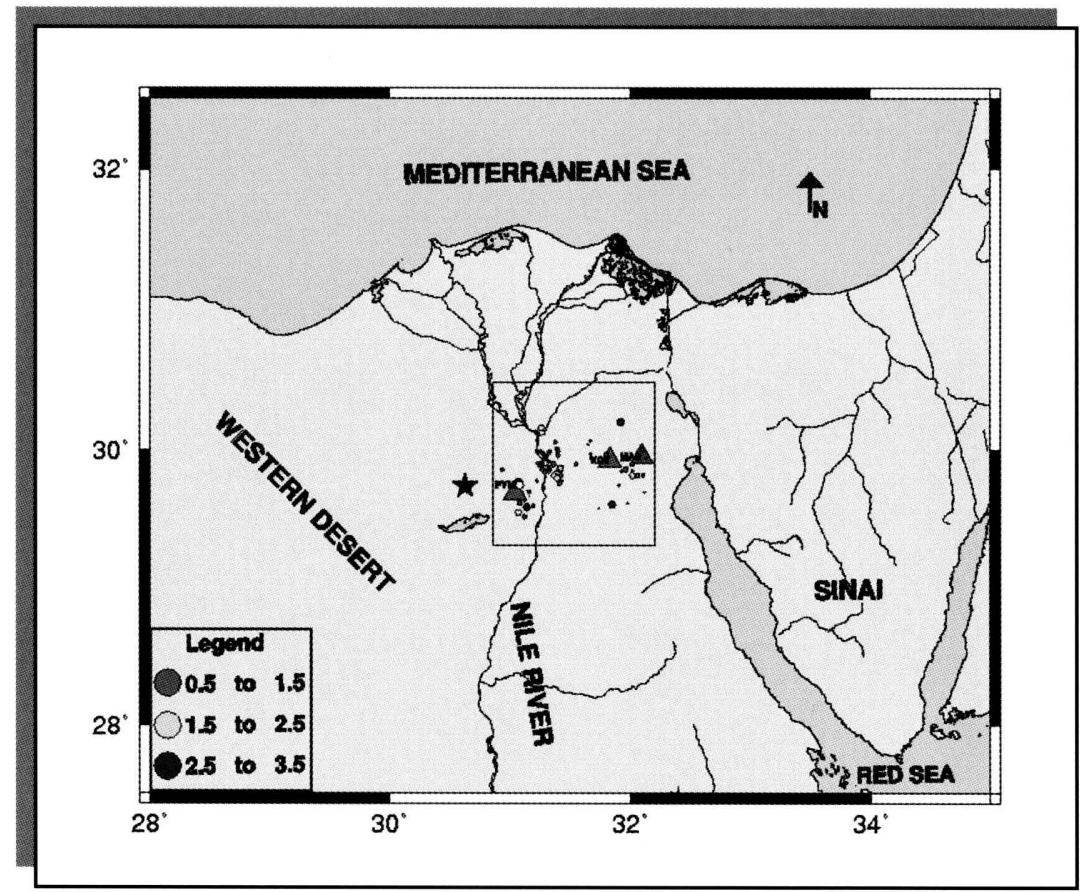

Fig. 2 Map showing the study area (solid square) and the locations of seismic stations (solid triangles) and events (circles) used in this study. Epicenter of the 1992 earthquake is also shown by a solid star.

\section{Method}

An important factor in studying the ground motion is the attenuation of seismic waves in their path between the source and the receiver. Several different approaches have been used to quantify the attenuation from different parts of seismograms (e.g., AKI, 1969, 1980; Herrmann, 1980; Mitchell, 1995), each of which could be sensitive to different physical processes (e.g., anelastic absorption and scattering). At local or regional distances $\left(<10^{\circ}\right)$, for evaluating the seismic wave attenuation of high frequencies (> $1 \mathrm{~Hz}$ ), the most common methods are as follows: (1) methods that parameterize the source and fit body wave spectra (e.g., Hough et al., 1988; Boatwright, 1978); (2) methods that cancel the seismic source through spectral ratio of different parts of seismograms (e.g., Frankel $e t$ al., 1990; AKı, 1980); (3) methods that measure coda amplitude decay with increasing lapse time (e.g., AKI and CHOUET, 1975); and (4) methods that use a nearby smaller event as an empirical Green's function (e.g., Hough, 1997). Some methods assume frequency-dependent $Q$, while others assume a 
frequency-independent $Q$. Historically, the study on the crustal seismic attenuation of $S$ waves has been more advanced than that on $P$ waves (CASTRO et al., 1990; KinOSHITA, 1994). It is mostly due to the requirement of the engineering seismology. The quality factor $(Q)$ determined using coda waves, hereafter called $Q_{c}$, has been used since the first measurements done by AKI and CHOUET (1975) as a crucial factor to describe the regional attenuation of seismic waves. This method has been used by several authors (e.g., MAMADA et al., 1997; NAKAMURA et al., 1997), and a comprehensive review was given by HERRAIZ and ESPINOSA (1987).

In order to estimate the $Q_{c}$ value, the single backscattering model (AKI and Chouet, 1975, SATo, 1977) was proposed in which coda waves are considered as backscattering waves coming from numerous scatterers (heterogeneities) distributed over a large area surrounding the stations and epicenters. If the seismic focus and the receiver are assumed to be at the same point and only the effect of single scattering is considered, the coda power spectrum $P(\omega \mid t)$ in 3-D media can be expressed as (AKI and CHOUET, 1975):

$$
P(\omega \mid t)=S(\omega) t^{-2} \mathrm{e}^{-\omega t / Q_{c}(\omega)}
$$

where $S(\omega)$ is a factor that describes the spectral characteristics of source, $\omega$ is the angular frequency of the wave under consideration, $t$ is the lapse time measured from the origin time. For seismograms of a certain frequency band, the relationship between the root mean square coda amplitude $A(\omega \mid t)$ and power spectrum is

$$
A(\omega \mid t)=[2 P(\omega \mid t) \Delta f]^{1 / 2}
$$

where $\Delta f$ is the band width. Combination of equations (1) and (2) gives

$$
\ln [A(\omega / t) t]=c(\omega)-\left[\omega / 2 Q_{c}(\omega)\right] t
$$

where $c(\omega)$ represents the source factor and band width, which is related only to frequency.

When coda records of a shorter lapse time are involved, the source and receiver must be considered as being not at the same point. According to the single isotropic scattering model (SATO, 1977), the energy density of scattered $S$ wave is then

$$
E(r, \omega \mid t)=\frac{W(\omega)}{4 \pi l r^{2}} K^{\prime}(\alpha)
$$

where $W$ is the total energy released by the source in unit angular frequency range, $l$ is the mean free path of $S$ wave, $r$ is the distance from the source to the point of receiver, $\alpha=t / t_{s}$ with $t_{s}$ being the travel time of $S$ wave, and the function $K^{\prime}(\alpha)$ is

$$
K^{\prime}(\alpha)=\frac{1}{\alpha} \ln \left(\frac{\alpha+1}{\alpha-1}\right) .
$$

For narrow-band displacement seismograms, there is the relationship

$$
E(r, \omega \mid t)=\rho \omega^{2} P(r, \omega \mid t)
$$

where $\rho$ is the density of medium. From equations (2), (4) and (6), the following equation can be derived:

$$
A(r, \omega \mid t)=\frac{1}{\omega}\left|\frac{W(\omega) \Delta f}{2 \pi \rho l}\right|^{1 / 2} \frac{\left|K^{\prime}(\alpha)\right|^{1 / 2}}{r} \mathrm{e}^{-\omega t / 2 Q_{c}(\omega)}=C^{\prime}(\omega) K(r, \alpha) \mathrm{e}^{-\omega t / 2 Q_{c}(\omega)},
$$


where $K(r, \alpha)=\left|K^{\prime}(\alpha)\right|^{1 / 2} / r$ is the modification factor of coda amplitude. The coda wave amplitude $A(\omega \mid t)$ at angular frequency $\omega$ and lapse time $t$ can then be expressed logarithmically as:

$$
\ln \frac{A(r, \omega \mid t)}{K(r, \alpha)}=C(\omega)-\frac{\omega}{2 Q_{c}(\omega)} t
$$

(AKI and CHOUET, 1975, SATO, 1977). $Q_{c}^{-1}$ is easily obtained by a linear regression as the slope of the straight line fitting the measured amplitude $A(\omega \mid t)$ versus lapse time $t$ at a fixed frequency $\omega$. The geometrical spreading is considered to be proportional to $r^{-1}$, which only applies to body waves in a uniform medium. The above equation has been validated in many studies, as summarized by HERRAIZ and ESPINOSA (1987).

$Q$-value usually varies with frequency $f$, and the form of variation can be taken as

$$
Q=Q_{0} f^{n}
$$

Besides, by actually observing the coda part, it can be seen that the decay rate of the coda envelope decreases with increasing lapse time. Many researchers (e. g., ZHANG, et al, 1991; Li, et al, 1991) have pointed out that the length of lapse time of coda is related to the reflected medium range or sampling depth. The starting time of coda window analyzed depends on the distance from the seismic source to the receiver. Empirically, it holds for $t>2 t_{s}$, where $t_{s}$ is the $S$-wave travel time. In order to obtain $A(\omega \mid t)$, the signal under analysis is bandpass filtered around $\omega$ (RAuTian and KHALturin, 1978). For analysis of the coda waves, we used the program developed by MAMADA and TAKENAKA (1997).

\section{Results}

In order to calculate the $Q$-values for seismic waves of different frequencies, it is necessary to use frequency division seismogram. We employ a narrow-band filter to obtain the frequency division seismograms from the original digital waveform records. Each trace of horizontal records was first filtered by using a phaseless Butterworth bandpass filter (Saito, 1978) with five passbands whose central frequencies are 1.5, 3.0, 6.0, 12 and $18 \mathrm{~Hz}$. An example of a bandpass-filtered seismogram used is shown in Figure 3.

Then, for determining $Q_{c}^{-1}$, a time window of $2(r / \beta)$ to $2(r / \beta)+20 \mathrm{sec}$ from the origin time of the earthquake was adopted to perform a least-squares regression of equation (8) for each frequency band. Two estimates of $Q_{c}^{-1}$ are obtained for each frequency band. One corresponds to NS component, while the other corresponds to $\mathrm{EW}$ component. On completion, the average values are calculated and a $Q_{c}^{-1}$ versus $f$ curve is fitted to the calculated values for each station, and then finally a general attenuation law was calculated from all data. The resulting $Q_{c}$ values for each station are shown in Figure 4 and Table 1.

Throughout the analysis conducted, no significant regional differences became evident, indicating that a very similar tectonic setting is being sampled by the coda waves. This result may justify the characterization of the entire region with a single attenuation relation. The regional $Q_{c}$ values obtained by using all station data are also shown in Figure 4 and Table 1. The regional attenuation law of the form

$$
Q_{c}=104.66 f^{0.79}
$$

was obtained. 


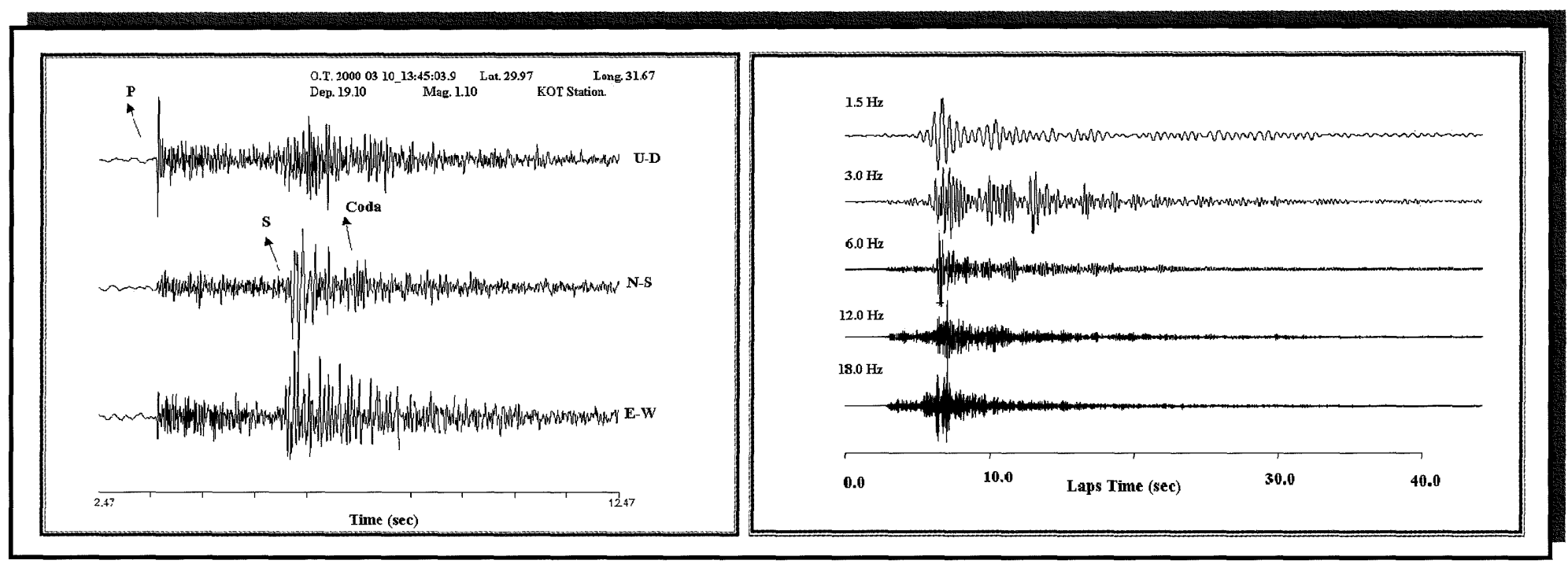

Fig. 3. Example of applying bandpass filtering to calculate $Q_{\mathrm{c}}$. Original three-component seismogram is shown in the left panel, while the bandpass filtered waveforms of the NS trace for five frequency bands are in the right panel. 


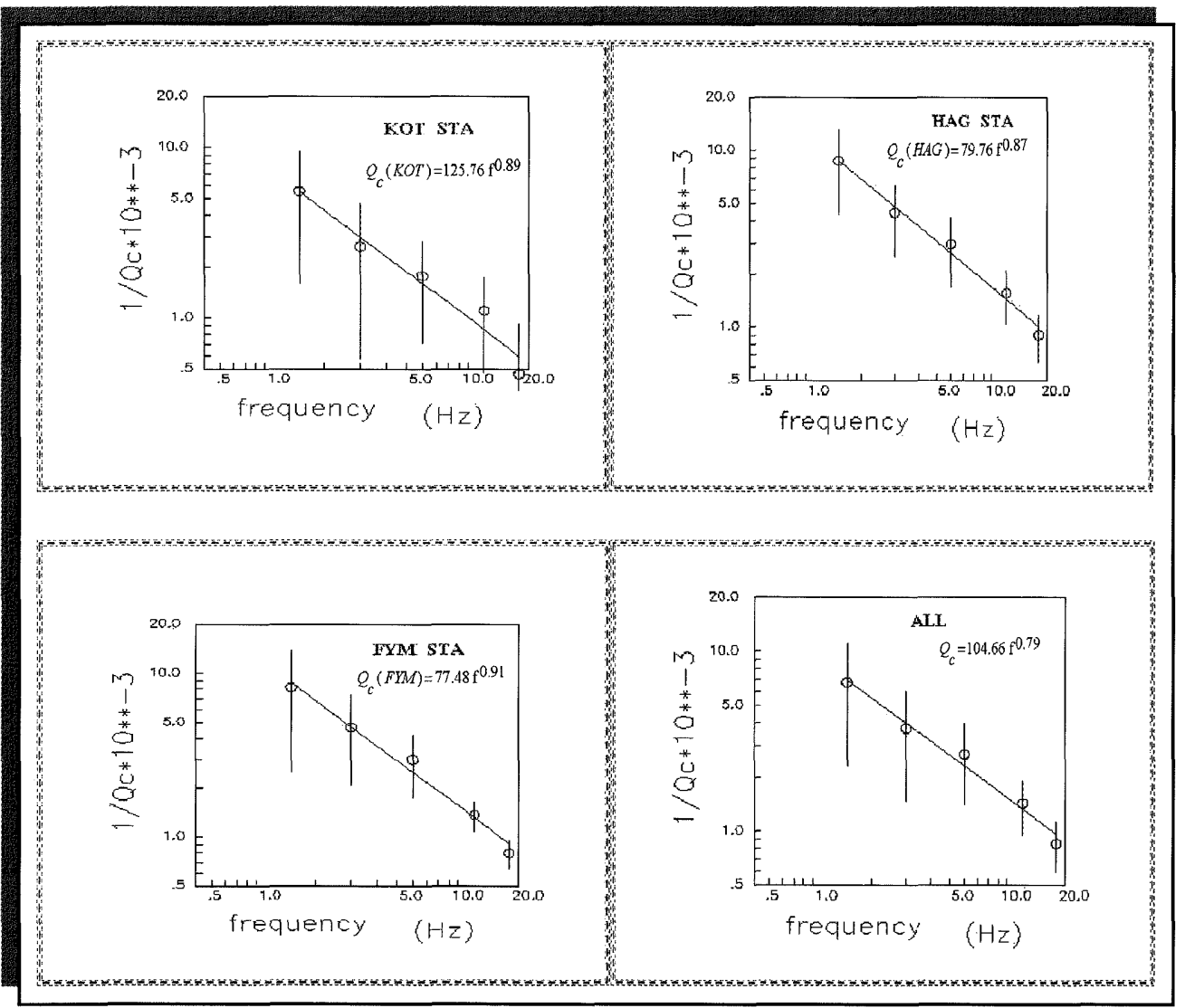

Fig. 4. The resulting $Q_{c}^{-1}$ values for each station and the general attenuation law from all stations. The solid line indicates the best-fit line from the least-squares estimate.

Table 1. Estimated $Q_{c}$ values for each station

\begin{tabular}{||c|c|c|c|c||}
\hline \hline$f(\mathbf{H z})$ & $\begin{array}{c}\text { FYM Station } \\
\mathbf{Q}_{\mathbf{c}}\end{array}$ & $\begin{array}{c}\text { HAG Station } \\
\mathbf{Q}_{\mathrm{c}}\end{array}$ & $\begin{array}{c}\text { KOT Station } \\
\mathbf{Q}_{\mathrm{c}}\end{array}$ & $\begin{array}{c}\text { ALL Stations } \\
\mathbf{Q}_{\mathbf{c}}\end{array}$ \\
\hline \hline 1.5 & 122.0075 & 114.4399 & 180.6026 & 148.5461 \\
3.0 & 213.7825 & 225.2304 & 382.8620 & 267.6308 \\
6.0 & 336.9474 & 339.0849 & 567.5929 & 374.5503 \\
12.0 & 734.0770 & 643.0217 & 910.5587 & 700.4926 \\
18.0 & 1256.4268 & 1108.7864 & 2143.1997 & 1173.1309 \\
\hline
\end{tabular}




\section{Discussion and conclusions}

The vicinity of Cairo Metropolitan Area has been studied in terms of coda wave attenuation. The single-scattering model of AKI and CHOUET (1975) has been applied to estimate $Q_{c}$. Considering that the selected seismogram should have the coda wave amplitude of a certain length of time on the one hand, and it should not be clipped on the other hand, the magnitude of events selected for analysis is $M_{\mathrm{L}}=0.7-3.5$. For earthquakes that the station network can give the epicenter, the seismograms recorded by the stations not farther than $40 \mathrm{~km}$ from the epicenter were selected. A total of 35 earthquakes have been analyzed, including 30 ones whose epicenters were given by the station network and five ones recorded by only a single station.

$Q_{c}$ is used to understand the regional attenuation of coda waves in the vicinity of Cairo area. $Q_{c}$ is calculated for groups of events and three stations at given frequencies. On completion, the average values were calculated and a $Q_{\mathrm{c}}^{-1}$ versus frequency curve was fitted to the calculated values. A regional attenuation law of the form $Q_{c}=104.67 f^{0.79}$ was obtained. Throughout the analysis conducted, no significant regional differences became evident, indicating that a very similar tectonic setting is being sampled by the coda waves. This result may justify the characterization of the entire region with a single attenuation relation. It is clear that, the generation mechanism of the coda wave $Q$-value is rather complex; it is related to the sampling range reflected, the components of different times of scattering and the regional tectonics. For this reason, the results obtained in this paper and the interpretation of them are only preliminary; verification by the computational results of data for other regions and theoretical explanation are still needed.

As pointed out in many studies, when discussing on seismic hazard analysis, the large uncertainties faced are not a defect of the methodologies implemented; they do result from the lack of knowledge about the earthquake phenomena. Besides, the quality of the results can be improved by obtaining and quantifying the appropriate inputs to the analysis. But, on the other hand, it is very important to understand the difference between reporting the extent of scientific uncertainty and the misunderstanding of the randomness of the phenomena.

This paper has attempted to understand a few basic phenomena related to the earthquake occurrence and the seismic hazard in Egypt. There is still a large amount of work to be done in order to improve the understanding of the behavior of seismic sources and their related effects in the country. Probably the three more relevant challenges for the earth scientists community in Cairo Metropolitan are:

(i) the improvement of the local seismic recording facilities,

(ii) the incorporation of multidisciplinary teams of experts into the study of the effects of the earthquake occurrence, and

(iii) the improvement in their relationship with the users of the earth science information.

\section{Acknowledgements}

Part of this work was done when the first author (S.S.R. M.) stayed in Kyushu University in April to July in 2001 for his individual study of the Seismology and Earthquake Engineering Training Course in the IISEE (International Institute of Seismology and Earthquake Engineering) sponsored by the JICA (Japan International Cooperation Agency). We are grateful to Dr Kenichi KATo of Kajima Corporation for fruitful discussion and Prof. Sadaomi Suzukı of Kyushu Univ. for carefully reading the manuscript and useful comments. 


\section{References}

AKI, K. (1969) Analysis of seismic coda of local earthquakes as scattered waves. J. Geophys. Res., 74, 615-631.

- (1980) Attenuation of shear-waves in the lithosphere for frequencies from 0.05 to $25 \mathrm{~Hz}$, Phys. Earth Planet. Inter., 21, 50-60.

and Chouet, B. (1975) Origin of coda waves: source attenuation and effects. J. Geophys. Res., 80, 3322-3342.

Boatwright, J. (1978) Detailed spectral analysis of two small New York State earthquakes, Bull. Seism. Soc. Am., 68, 1117-1131.

CASTRO, R.R., ANDERSON, J.G. and Singh, S.K. (1990) Site response, attenuation and source spectra of S waves along the Guerrero, Mexico, subduction zone. Bull. Seism. Soc. Am., 80, 1481-1503.

Frankel, A., McGarr, A., Bicknell, J., Mori, J., Seeber, L., and Cranswick, E. (1990) Attenuation of high-frequency shear waves in the crust : Measurements from New York State, South Africa, and Southern California, J. Geophys. Res., 95, 17441-17457.

Herraiz, M. and EspinosA, A.F. (1987) Coda waves: A review. Pure Appl. Geophys., 125, 499-577.

Herrmann, R. (1980) $Q$ estimates using the coda of local earthquakes, Bull. Seism. Soc. Am., 70, 447468.

Hough, S., Anderson, J. G., Brune, J., Vernon, F., Berger, J., Fletcher, J.,HaAr, L., Hanks, T., and Baker, L. (1988) Attenuation near Anza, California. Bull. Seism. Soc. Am..78, 672-691.

Hough, S. E. (1997) Empirical Green's function analysis : Taking the next step. J. Geophys. Res., 102, 5369-5384.

KInoshitA, S. (1994) Frequency-dependent attenuation of shear waves in the crust of the Southern Kanto area, Japan. Bull. Seism. Soc. Am., 84, 1387-1396.

Li SONG-Lin, FAN Ji-CHANG, Hui NAI-Ling, et al. (1991) A study on coda $Q$-value and its relationship with frequency of the Luanxian region,China. Acta Seismologica Sinica, 4(4): 509-520.

Mamada, Y., Okumura, T., Takenaka, H., Suzuki, S., Saito, S., Furumura, M., Sasatani, T., Matsushima, T. and Furumura, T. (1997) Coda $Q^{-1}$ in Awaji island estimated from the aftershock records of the 1995 Hyogo-ken Nanbu earthquake. J. Phys. Earth, 45, 147-154.

- and TAKENAKA, H. (1997) Comparison of $Q_{c}^{-1}$ estimates from coda envelopes constructed from one- and multi-component seismograms. J. Phys. Earth, 45, 455-466.

Mitchell, B. (1995) Anelastic Structure and Evolution of the Continental Crust and Upper Mantle from Seismic Surface Wave Attenuation, Rev. Geophys., 33, 441-462.

Nakamura, S., Kubo, A., OhKura, T. and Ouchi, T. (1997) Attenuation of seismic waves along the Rokko fault zone. Zisin (J. Seis. Soc. Japan), 50, 173-82 (in Japanese with English abstract).

Rautian, T.G. and Khalturin, V.I. (1978) The use of the coda for determination of the earthquake source spectrum. Bull. Seism. Soc. Am., 68, 923-948.

SAITO, M. (1978) An automatic design algorithm for band selective recursive digital filters. Geophysical Exploration (Butsuri-Tanko), 31, 112-135 (in Japanese with English abstract)

SATO, H. (1977) Energy propagation including scattering effects, single isotropic scattering approximation. J. Phys. Earth, 25, 27-41.

Zhang Tian-Zhong, Gao Long-sheng, Zhang Wei-ping. (1991) The $Q$-factor and its time window dependence in the area of Western-Yunnan Seismological Experiment Site [J]. Acta Seismologica Sinica, 4(1), 15-28. 